\title{
Food and drink consumption among 1-5-year-old Los Angeles County children from households receiving dual SNAP and WIC v. only WIC benefits
}

\author{
Jane Liu ${ }^{1,2}$, Tony Kuo ${ }^{1,2,3, *}$, Lu Jiang ${ }^{4}$, Brenda Robles ${ }^{2,5}$ and Shannon E Whaley ${ }^{4}$ \\ 'Department of Epidemiology, UCLA Fielding School of Public Health, Los Angeles, CA, USA: ${ }^{2}$ Division of Chronic \\ Disease and Injury Prevention, Los Angeles County Department of Public Health, 3530 Wilshire Blvd, \\ 8th Floor, Los Angeles, CA 90010, USA: ${ }^{3}$ Department of Family Medicine, David Geffen School of Medicine at \\ UCLA, Los Angeles, CA, USA: ${ }^{4}$ Public Health Foundation Enterprises WIC Program, Irwindale, CA, USA: \\ ${ }^{5}$ Department of Community Health Sciences, UCLA Fielding School of Public Health, Los Angeles, CA, USA
}

Submitted 30 December 2015: Final revision received 2 July 2016: Accepted 11 July 2016: First published online 9 September 2016

\begin{abstract}
Objective: The Supplemental Nutrition Assistance Program (SNAP) and the Special Supplemental Nutrition Program for Women, Infants, and Children (WIC) are two of the more well-known food assistance programmes in the USA. The current study describes food consumption patterns of children aged 1-5 years living in households dually enrolled in these two programmes $v$. households enrolled only in WIC.

Design: Food consumption and SNAP participation were assessed using data from the 2014 Survey of Los Angeles County (LAC) WIC Participants and the Follow-Up Survey of the same households that were also SNAP beneficiaries. Telephone interviews were conducted with WIC parents regarding each child's (i.e. beneficiary's) food consumption patterns. Follow-up interviews were conducted with those who reported receiving SNAP. Multivariable regression analyses were performed to assess the relationships between food and beverage consumption and dual $v$. single food assistance programme participation.

Setting: LAC, California.

Subjects: Children of WIC-enrolled households in LAC during 2014 ( $n$ 3248). This included a sub-sample of dual WIC- and SNAP-enrolled households ( $n$ 1295). Survey participants were the beneficiaries' parents.

Results: Children from dually enrolled households consumed $1.03(P<0.05)$ and $1.04(P<0.01)$ more servings of fruits and vegetables daily respectively, 1.07 more sugar-sweetened beverages daily $(P<0.001)$ and ate sweets/sweetened foods 1.04 more times daily $(P<0.001)$ than children from households participating only in WIC. Conclusions: Results suggest that SNAP+WIC enrolment is associated with increased consumption of both healthy foods and foods containing minimal nutritional value. Complementary nutrition education efforts across the two programmes may help beneficiaries maximize healthful food purchases with SNAP dollars.
\end{abstract}

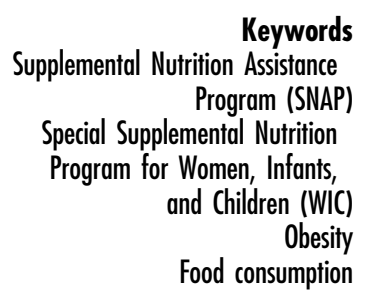

The Supplemental Nutrition Assistance Program (SNAP) and the Special Supplemental Nutrition Program for Women, Infants, and Children (WIC) comprise two of the more wellknown food assistance programmes in the USA. The primary goal of these federally funded programmes is to increase food access and the purchasing power of low-income families living at or below the federal poverty level ${ }^{(1)}$.

SNAP (previously called the Food Stamp Program; 'CalFresh' in California) aims to prevent hunger and provide nutritional resources to households with monthly gross incomes at or below $200 \%$ of the federal poverty level (exceptions include households with elderly or disabled individuals $)^{(2-5)}$. The WIC programme is designed to promote nutrition quality and overall health in a more targeted population: pregnant and postpartum women, and children aged $0-5$ years. WIC serves families with annual incomes at or below $185 \%$ of the federal poverty level ${ }^{(6)}$.

In spite of sharing a similar mission, these two food assistance programmes differ in a number of ways, most notably on how beneficiaries (members of households) can utilize programme benefits. SNAP households, for example, can use benefits to purchase a wide variety of food items, including foods with minimal nutritional value $^{(3)}$. Beneficiaries typically receive electronic benefit 
transfer cards for food purchases at participating grocery stores. In contrast, WIC restricts purchases to specific supplemental foods - defined in the literature as foods that are lacking in the diets of low-income women and children. In California, WIC benefits are issued on paper vouchers and redeemed at authorized stores. WIC-eligible foods include milk, cheese, eggs, wholegrain products, fruits and vegetables, and other healthy foods that contribute nutrients found to be lacking in the diets of low-income, pregnant and postpartum women, infants and children up to age 5 years $^{(6)}$. Unlike SNAP, WIC guidelines prohibit the purchase of foods with minimal nutritional value.

In addition to receiving food benefits, WIC participants are also provided nutrition education at least four times annually $^{(7)}$. These education services are a core service of the WIC programme and are delivered to beneficiaries at WIC sites staffed by nutrition professionals and paraprofessionals. The services are delivered 1:1 or in a group setting. In recent years, alternative modes of delivery such as Internet-based education have been developed and are increasingly available to WIC parents ${ }^{(7)}$.

SNAP households are also eligible to receive nutrition education $^{(8-10)}$; albeit the content of the education is often dissimilar to WIC. In 2010, it was estimated that there were about two million households in Los Angeles County that were eligible for this education service ${ }^{(8)}$. Known as 'Champions for Change', SNAP nutrition education ('SNAP-Ed') proactively targets eligible populations regardless of actual enrolment ${ }^{(11)}$. Unlike WIC, receiving SNAP-Ed services is not mandatory and does not require confirmation of SNAP enrolment.

In California and elsewhere in the USA, low-income families can enrol in both SNAP and WIC simultaneously depending on their income status. To date, only a few health research studies have examined this multi-eligibility phenomenon, specifically multi-eligibility for SNAP and WIC, and its connection(s) to eating behaviours ${ }^{(12-19)}$. Similarly, only one economic study was found that focused on the economic impact of both SNAP and WIC on food intake ${ }^{(20)}$. Current US census data indicate that $42 \%$ of low-income families participate in only one food assistance programme while $22 \%$ participate in at least two out of the four major food assistance programmes supported by the US Department of Agriculture: SNAP, WIC, free school meals and reduced-price school meals ${ }^{(12)}$. We sought to address these and other gaps about food assistance programmes in the literature by describing the characteristics and health-related behaviours of children aged $1-5$ years living in households receiving SNAP and WIC $v$. households receiving only WIC benefits.

\section{Methods}

\section{Study design and population}

The present study utilizes data from the 2014 Survey of Los Angeles County WIC Participants ${ }^{(21)}$ and the supplemental
Follow-Up Survey of households that were also SNAP beneficiaries. The study explores the potential relationships between child food (e.g. fruits and vegetables) and drink (e.g. sugar-sweetened beverages (SSB)) consumption and dual $v$. single programme participation. In the present study, fruit and vegetable consumption was used as a proxy to assess each child's (beneficiary's) frequency of eating healthfully ${ }^{(22,23)}$. SSB consumption and consumption of sweets were proxies to gauge each child's intake of foods with minimal nutritional value. Because Los Angeles County is divided into eight Service Planning Areas, the cross-sectional study sample was drawn from all eight regions. To account for the more sparsely populated areas of Los Angeles County, Service Planning Area 1 - Antelope Valley was oversampled ${ }^{(24)}$.

The triennial WIC household survey was adapted from the 2005 Los Angeles County Health Survey ${ }^{(25)}$ with extensive input from the California State WIC Division and the WIC Local Agency staff. The primary objective of the serial WIC Survey was to assess key health indicators and health-related behaviours as well as home and community environments of WIC families living in Los Angeles County. In 2014, a supplemental follow-up survey was developed by the Los Angeles County Department of Public Health and carried out to assess similar indicators among WIC families also receiving SNAP benefits ${ }^{(24)}$.

For both surveys, telephone interviews were administered in either English or Spanish (based on the language preference of each participant) to WIC parents who were representing the child beneficiary. Calls were made to 12026 Los Angeles County WIC participants with a valid telephone number. Of them, 6008 completed the survey ( $50 \%$ response rate). Of those whom interviewers were able to reach by telephone, $88 \%$ agreed to participate in the survey ( $88 \%$ cooperation rate). Four thousand nine hundred and ninety of the 6008 participants constitute the main WIC Survey sample for the current study (the remaining 1018 belonged to a special augment sample) ${ }^{(26)}$. The survey questionnaires were initially developed in English and later translated into Spanish. Both versions were piloted with prospective participants to ensure clarity and consistency. Approval from the Independent Review Consulting Institutional Review Board that served the Public Health Foundation Enterprises WIC Program was obtained for all protocols used in the main survey prior to field implementation. For the Follow-Up Survey, the Los Angeles County Department of Public Health Institutional Review Board also reviewed and approved the protocols. All interviews were conducted by the Field Research Corporation - an independent public opinion survey firm - using a computerassisted telephone interviewing system. Participants in the main survey were asked for permission to be re-contacted for a follow-up survey pertaining to SNAP. Only parents in WIC households who were identified as receiving SNAP benefits in the last 12 months and who gave their consent 
to be re-contacted were eligible and were called for the follow-up survey.

\section{Regression analysis measures}

Dependent variables: child food and drink consumption To measure healthy food or drink consumption, parents of each child beneficiary were asked the following questions: (i) 'On an average day, how many servings of fruits does NAME eat?'; (ii) 'On an average day, how many servings of vegetables does NAME eat?'; (iii) 'On an average day, how many times does NAME drink water?'; (iv) 'On an average day, how many times does NAME drink milk?'; and (v) 'If Q32e >0, ask: What kind of milk does NAME most drink?' The two questions pertaining to milk, (iv) and (v), were combined and coded into a new milk variable, which was used as the continuous variable for the number of times per day a child drank whole, $1 \%, 2 \%$ or non-fat milk.

To measure consumption of foods or drinks with minimal nutritional value, parents were asked the following questions: (vi) 'On an average day, how many times does NAME eat sweets or sweetened foods, such as sweetened cereals, fruit bars, pop-tarts, donuts, cookies and candies?'; (vii) 'On an average day, how many other fruit juice drinks that are not $100 \%$ juice, such as Sunny Delight, Capri Sun, or Lemonade does NAME drink?'; (viii) 'On an average day, how many sweetened drinks such as Gatorade, Kool Aid, or Red Bull does NAME drink?'; and (ix) 'On an average day, about how many regular sodas such as Coke or Mountain Dew, does NAME drink? Do not include diet sodas or sugar-free drinks.' For all nutrition-related consumption questions, parents were given the option of indicating 'don't know' or 'refused.' For questions (vii), (viii) and (ix), parents were also given the option of indicating 'some but less than 1 serving'. The number of SSB a child consumed daily was determined through combining questions (vii), (viii) and (ix) into a single variable. All food or drink variables were analysed as continuous variables. Responses of 'don't know' or 'refused' were excluded from the analysis (set to missing) and responses of 'some but less than 1 serving' for questions (vii), (viii) and (ix) were set to $0 \cdot 25$ servings.

Independent variables: sociodemographic variables and programme participation status

All survey participants in the sample were WIC parents who were the primary caregiver of the beneficiaries (WIC children). Participants were identified as receiving SNAP benefits if they answered 'yes' to the question: 'In the last twelve months, have you or has anyone in your household used an EBT [electronic benefit transfer] card for food stamps to buy food?' A categorical variable with two groups was generated to distinguish those who replied 'yes' (dual SNAP and WIC group) $v$. those who replied 'no' (only WIC group).
The sociodemographic variables chosen as covariates for the study analyses included: (i) language of interview (English or Spanish); (ii) parent education (less than high school $v$. high school graduate or higher); (iii) child age (continuous variable); and (iv) child gender (male or female). Race/ethnicity was excluded as a covariate due to the high density of Hispanic participants in the sample (over $80 \%$ ) and language of survey was included to adjust for differences among the Hispanic populations. From the original data set codebook, 'Hispanic - English' and 'Hispanic - Spanish' were formatted as a single 'Hispanic' ethnicity label. Parent education was dichotomized into a binary variable by combining 'no schooling', '8th grade or less' and 'grade 9-12' into a category labelled 'less than high school', and combining 'high school grad', 'some college associate degree', 'college grad' and 'post grad' into a category labelled 'high school graduate or higher'. Other demographic variables remained unchanged from their original formats.

Independent variables: parent perceptions of their child's weight status and parent report of housebold food security Parental perception of child weight status was included as an independent variable in order to adjust for the potential influence of parental perception of child's health on food choices parents make for their children. To evaluate parent perceptions of their child's weight status, parents were asked, 'Right now, do you consider NAME to be overweight, underweight or about right for (his)/(her) height?' Parents were then given the option to indicate 'overweight', 'underweight', 'about right', 'don't know' or 'refused'. Parental perception of child weight status was operationalized as a categorical variable ('overweight', 'underweight' or 'about right'). Parental perception of their household food security status was included as a covariate to control for the potential effects of parental attitudes towards their family's food security on programme participation and on food consumption behaviour. To assess parental perception of their families' food security status in the past 12 months, parents were presented with the US Department of Agriculture's six-item US Household Food Security Survey Module ${ }^{(27)}$.

\section{Data analysis}

Descriptive and univariate analyses were conducted to describe the sociodemographic characteristics, parent perceptions, and food and drink consumption patterns of children from households receiving SNAP and WIC $v$. only WIC benefits. Only complete cases were included in the regression analyses. Demographics and parent perception variables were analysed and reported as categorical variables, while nutrition-related consumption variables were analysed as continuous variables (through $\log$ transformation) and reported as mean values with corresponding SD. 
A series of multivariable linear regression analyses was performed to assess the relationships between each continuous dependent variable (fruit and vegetable consumption, water, milk products, SSB, and sweets or sweetened foods) and food assistance programme participation status, controlling for sociodemographics and parent perception variables. This choice of modelling is a widely used method for evaluating the direction and strength of associations between multiple factors and a continuous outcome variable ${ }^{(28)}$. Literature examining correlates of fruit and vegetable consumption among adults and children ${ }^{(15,23,29)}$ informed covariate selection for the models. Due to the skewness in the upper tail of the distributions for each continuous outcome variable (fruits, vegetables, water, milk, SSB, sweets/sweetened foods), the continuous outcome variables were log transformed using $\log (x+1)$, where $x$ is the dependent variable, in order to analyse the variable as a normal distribution and to prevent data loss. The log-transformed parameter estimates that resulted from the model were retransformed through exponentiation to produce the final linear model describing the relationships between the continuous dependent variables and the independent variables. A total of five models were constructed using one of five dependent variables as the outcome for each model. Each model controlled for the same covariates. All data for the present study were cleaned and analysed using the statistical software package SAS version 9.4.

\section{Results}

The 2014 Survey of Los Angeles County WIC Participants contacted a total of 4990 households. The survey comprised a main cross-sectional sample of 4691 participants randomly selected from the population of all WIC households (families) receiving services in Los Angeles County during January 2014; an additional 299 participants from Antelope Valley (i.e. Los Angeles County's least populous region) were recruited for a total sample of 4990. A total of 2359 households were identified as having received SNAP benefits during the past 12 months; of these, 1605 WIC households were found to be additionally enrolled in SNAP over the past year. As a result, the 1605 dually enrolled parents were re-contacted and completed the Follow-Up Survey interview. The response rate for this follow-up survey was $68 \%$. Changed/disconnected/ incorrect number or reaching the answering machine represented the primary reasons for non-response. Among the combined sample (both surveys), 1605 households were identified as receiving both SNAP and WIC benefits in the last 12 months and 2629 were identified as receiving only WIC benefits.

Among the combined sample of dually enrolled SNAP and WIC and only WIC households, a majority of the parents (86\%) were Hispanic and about $64 \%$ graduated from high school or higher. Among the children of dually enrolled households, $85 \%$ were Hispanic, $51 \%$ were male, and the average age was about $2 \cdot 5$ years (Table 1 ).

Children from both groups (dually enrolled and only WIC enrolled) were found to consume approximately five servings of fruits and vegetables daily, drank water about four times daily and drank milk products about twice daily (Table 2). Children from households receiving both WIC and SNAP also consumed slightly more sweets or sweetened foods daily than children from households receiving only WIC; this was true for consumption of non-diet SSB as well (Table 3).

In multivariable regression analyses, children from households receiving SNAP and WIC benefits were found to consume 1.03 more servings of fruits daily $(P<0.05)$, 1.04 more servings of vegetables daily $(P<0.01), 1.07$ more non-diet SSB daily $(P<0 \cdot 001)$ and ate sweets or sweetened foods 1.04 more times daily $(P<0.001)$ than children from households receiving only WIC benefits (Table 3). No statistically significant differences in water or milk product consumption by programme participation status were found in the analyses.

\section{Discussion}

The current study found that children aged $1-5$ years from households receiving SNAP and WIC consumed more servings of fruits and vegetables daily than children from households receiving only WIC benefits. Prior health research has focused primarily on the impact of single food assistance programme enrolment on healthful food consumption rather than on dual enrolment in SNAP and WIC. For example, a study on diets of pre-school children discovered that participants of WIC had a higher prevalence of fruit consumption compared with participants who were eligible but not participating in the programme ${ }^{(30)}$. The literature on SNAP-Ed suggests a similar pattern. In an analysis to assess the effects of SNAPEd services, researchers found that households receiving 'high reach' nutrition education interventions reported more fruit and vegetable consumption than households that did not receive these interventions ${ }^{(31)}$. Moreover, prior research on the economic implications of multiple food assistance programme participation indicates that receiving higher food assistance benefits alone may not be sufficient to promote healthy food consumption ${ }^{(32,33)}$. Additional subsidies only increased healthy food purchases when the subsidies were specifically created for the purchase of fruits and vegetables, indicating that increased subsidies from multiple food assistance programmes as compared with one or no food assistance may not be the sole indicator for more purchase of healthy foods. In a previous study on the economic impact of SNAP and WIC participation on fruit and vegetable intake in food desert communities $v$. non-food desert 
Table 1 Comparison of sociodemographic characteristics between households with SNAP and WIC $v$. only WIC benefits; results from the 2014 Survey of Los Angeles County WIC Participants and the Follow-Up Survey, 2014

\begin{tabular}{|c|c|c|c|c|c|c|}
\hline & \multicolumn{2}{|c|}{$\begin{array}{l}\text { Overall sample: main and follow-up } \\
\text { surveys combined ( } n \text { 3248) }\end{array}$} & \multicolumn{2}{|c|}{$\begin{array}{l}\text { SNAP and WIC household } \\
\text { sample }(n \text { 1295) }\end{array}$} & \multicolumn{2}{|c|}{$\begin{array}{l}\text { Only WIC household } \\
\text { sample }(n \text { 1953) }\end{array}$} \\
\hline & $n$ or Mean & $\%$ or SD & $n$ or Mean & $\%$ or SD & $n$ or Mean & $\%$ or SD \\
\hline Total, $n$ and \% & $3248 \dagger$ & 100 & $1295 \dagger$ & 100 & $1953 \dagger$ & 100 \\
\hline \multicolumn{7}{|l|}{ Parent characteristics } \\
\hline \multicolumn{7}{|l|}{ Race/ethnicity, $n$ and \% } \\
\hline Hispanic & 2800 & $86 \cdot 3$ & 1075 & $83 \cdot 1$ & 1725 & 88.4 \\
\hline Non-Hispanic White & 131 & 4.0 & 52 & 4.0 & 79 & $4 \cdot 1$ \\
\hline Non-Hispanic Black & 211 & 6.5 & 127 & $9 \cdot 8$ & 84 & 4.3 \\
\hline Non-Hispanic Asian/Pacific Islander $\ddagger$ & 75 & $2 \cdot 3$ & 21 & 1.6 & 54 & $2 \cdot 8$ \\
\hline Other & 27 & 0.8 & 18 & 1.4 & 9 & 0.5 \\
\hline \multicolumn{7}{|l|}{ Education, $n$ and \% } \\
\hline Less than high school & 1168 & $36 \cdot 1$ & 593 & $46 \cdot 0$ & 575 & $29 \cdot 6$ \\
\hline High school graduate or higher & 2065 & 63.9 & 696 & $54 \cdot 0$ & 1369 & $70 \cdot 4$ \\
\hline \multicolumn{7}{|l|}{ Language of interview, $n$ and \% } \\
\hline English & 1634 & $50 \cdot 3$ & 609 & $47 \cdot 0$ & 1025 & 52.5 \\
\hline Spanish & 1614 & $47 \cdot 0$ & 686 & 53.0 & 928 & 47.5 \\
\hline \multicolumn{7}{|l|}{ Perception of family food security, $n$ and \% } \\
\hline High or marginal food security & 2222 & 68.4 & 764 & 59.0 & 1458 & 74.7 \\
\hline Low food security & 720 & $22 \cdot 2$ & 362 & 28.0 & 358 & $18 \cdot 3$ \\
\hline Very low food security & 306 & $9 \cdot 4$ & 169 & $13 \cdot 1$ & 137 & $7 \cdot 0$ \\
\hline \multicolumn{7}{|l|}{ Child characteristics } \\
\hline \multicolumn{7}{|l|}{ Gender, $n$ and \% } \\
\hline Female & 1571 & 47.4 & 638 & $49 \cdot 3$ & 933 & $47 \cdot 8$ \\
\hline Male & 1677 & 51.6 & 657 & $50 \cdot 7$ & 1020 & $52 \cdot 2$ \\
\hline \multicolumn{7}{|l|}{ Race/ethnicity, $n$ and \% } \\
\hline Hispanic & 2845 & 87.9 & 1096 & $85 \cdot 1$ & 1749 & 89.7 \\
\hline Non-Hispanic White & 106 & $3 \cdot 3$ & 45 & 3.5 & 61 & $3 \cdot 1$ \\
\hline Non-Hispanic Black & 187 & $5 \cdot 8$ & 111 & 8.6 & 76 & 3.9 \\
\hline Non-Hispanic Asian/Pacific Islander & 60 & 1.9 & 13 & 1.0 & 47 & 2.4 \\
\hline Other & 40 & $1 \cdot 2$ & 23 & 1.8 & 17 & 0.9 \\
\hline \multicolumn{7}{|c|}{ Parent perception of child weight status, $n$ and $\%$} \\
\hline Overweight & 127 & 3.9 & 54 & 4.2 & 73 & $3 \cdot 7$ \\
\hline Underweight & 120 & 3.7 & 45 & 3.5 & 75 & $3 \cdot 8$ \\
\hline About right & 2998 & 92.4 & 1195 & 92.4 & 1803 & 92.4 \\
\hline Age (years), mean and SD & 2.95 & $1 \cdot 19$ & 2.47 & 1.45 & $2 \cdot 30$ & 1.51 \\
\hline
\end{tabular}

SNAP, Supplemental Nutrition Assistance Program; WIC, Special Supplemental Nutrition Program for Women, Infants, and Children.

Summary frequency analyses were used to derive the above values. Some variables contain missing data and may not add up to $100 \%$. 'Don't know'/'refused' responses were set to missing.

†Analysis limited to child age range 1-5 years for purposes of this analysis.

flncludes those self-identifying as Asians or as Pacific Islanders.

Table 2 Comparison of child food and drink consumption characteristics between households with WIC and SNAP V. only WIC benefits; results from the 2014 Survey of Los Angeles County WIC Participants and the Follow-Up Survey, 2014

\begin{tabular}{|c|c|c|c|c|c|c|}
\hline & \multicolumn{2}{|c|}{$\begin{array}{l}\text { Overall sample: main and } \\
\text { follow-up surveys combined } \\
(n 3248)\end{array}$} & \multicolumn{2}{|c|}{$\begin{array}{l}\text { SNAP and WIC household } \\
\text { sample }(n \text { 1295) } \\
\end{array}$} & \multicolumn{2}{|c|}{$\begin{array}{l}\text { Only WIC household } \\
\text { sample ( } n \text { 1953) }\end{array}$} \\
\hline & $n$ or Mean & $\%$ or SD & $n$ or Mean & $\%$ or SD & $n$ or Mean & $\%$ or $\mathrm{sL}$ \\
\hline Total, $n$ and $\%$ & 3248 & 100 & 1295 & 100 & 1953 & 100 \\
\hline \multicolumn{7}{|l|}{ Child food consumption characteristics, mean and SD } \\
\hline Non-diet SSB (no. of drinks/d)† & 0.78 & $1 \cdot 22$ & 0.91 & $1 \cdot 32$ & 0.69 & $1 \cdot 15$ \\
\hline Fruits (no. of servings/d) & 3.05 & 1.41 & 3.05 & 1.25 & 3.05 & 1.50 \\
\hline Vegetables (no. of servings/d) & $2 \cdot 27$ & $1 \cdot 24$ & $2 \cdot 30$ & $1 \cdot 32$ & $2 \cdot 25$ & $1 \cdot 19$ \\
\hline Water (no. of times/d) & 4.27 & 2.88 & $4 \cdot 27$ & 2.98 & 4.27 & $2 \cdot 81$ \\
\hline Milk - whole, $1 \%, 2 \%$ or non-fat (no. of times/d) & $2 \cdot 28$ & $4 \cdot 17$ & $2 \cdot 30$ & 1.47 & $2 \cdot 27$ & 1.37 \\
\hline $\begin{array}{l}\text { Sweets or sweetened foods, such as sweetened } \\
\text { cereals, fruit bars, pop-tarts, doughnuts, cookies } \\
\text { and candies (no. of times/d) }\end{array}$ & 0.85 & 0.78 & 0.90 & 0.82 & 0.81 & 0.75 \\
\hline
\end{tabular}

SNAP, Supplemental Nutrition Assistance Program; WIC, Special Supplemental Nutrition Program for Women, Infants, and Children; SSB, sugar-sweetened beverages.

Summary frequency analyses were used to derive the above values. Some values may not add up to $100 \%$ due to missing data. 'Don't know' or 'refused' were excluded from the study analysis.

†'Some but less than 1 full serving' was set to 0.25 servings. 
Table 3 Predictors of child food and drink consumption patterns between households with SNAP and WIC $v$. only WIC benefits; results from the 2014 Survey of Los Angeles County WIC Participants and the Follow-Up Survey, 2014

PRIMARY REGRESSOR: Food assistance programme participation status (ref.: only WIC)†

\begin{tabular}{lc}
\hline & $\begin{array}{c}\text { Log transformed } \\
\text { coefficient }\end{array}$ \\
\hline MODEL DEPENDENT VARIABLE: Consumption & \\
Model 1B: Fruits (no. of servings/d), $n 3734$ & $1.03^{\star}$ \\
Model 1C: Vegetables (no. of servings/d), & $1.04^{\star *}$ \\
$n$ 3760 & 1.03 \\
Model 2: Water (no. of times/d), $n 3710$ & -1.02 \\
Model 3: Milk - whole, $1 \%, 2 \%$ or non-fat \\
(no. of times/d), $n$ 3178 & $1.07^{\star * *}$ \\
Model 4: Non-diet SSB (no. of drinks/d), & \\
$n$ 3793 & $1.04^{\star * *}$ \\
Model 5: Sweets or sweetened foods, such \\
as sweetened cereals, fruit bars, pop-tarts, \\
doughnuts, cookies and candies (no. of \\
times/d), $n 4188$
\end{tabular}

SNAP, Supplemental Nutrition Assistance Program; WIC, Special Supplemental Nutrition Program for Women, Infants, and Children; ref., reference category; SSB, sugar-sweetened beverages.

Independent variables used as controls for each model were language of interview, parent education, child age, child gender, parental report of household (family) food security and parental perception of child's weight status.

${ }^{\star} P<0.05,{ }^{\star \star} P<0.01,{ }^{\star * \star} P<0.001$.

†Only survey participants without missing data (including 'don't know'/ 'refused') for selected variables were included in the multivariable analyses. $\ddagger$ Significance level ( $P$ value) was derived using log transformation of the parameter estimate; log transformation was performed due to the skewness of the parameter data.

communities, researchers found a positive association between SNAP/WIC participation and increased fruit and vegetable consumption ${ }^{(20)}$. Potentially, because WIC's food packages indirectly subsidize the purchase of healthy foods, those who also receive SNAP may utilize the additional funds to purchase greater quantities of WIC package foods. A prior systematic review reinforces this hypothesis, showing that subsidization of specific foods through subsidies for those items has important influences over food choice among programme beneficiaries ${ }^{(33)}$.

The present study adds to the existing body of literature by showing the potential for dual programme enrolment to further increase fruit and vegetable consumption among food assistance programme beneficiaries. However, further research is needed to better understand the relationship between food subsidization and healthy food choice, given the unintended increases in specific unhealthy food items (e.g. sweets and SSB) along with healthy foods (e.g. fruits and vegetables) found in the present study.

Although some experts have suggested that WIC's food package and its restrictions to healthy foods may produce a 'crossover' effect on beneficiaries who also receive SNAP, the literature has not been able to fully validate this hypothesized programme outcome. Non-WIC data suggest that low-income populations tended to have less access to fruits and vegetables and were less likely to consume them $^{(34)}$, usually as a result of cost constraints and/or a lack of availability to fresh produce (e.g. no nearby grocery stores, no farmers' markets, concerns over neighbourhood safety) ${ }^{(35-37)}$, and not necessarily as a result of limited nutrition education or food assistance programme restrictions. In the present study, the increase in fruit and vegetable consumption among beneficiaries with dual SNAP and WIC affirms in part the emerging theories on how the WIC food package affects food selection behaviours in programme participants. The observed relationship, however, is ecological in nature and is tempered by the second finding of the study: children from households receiving SNAP and WIC also consumed a greater quantity of foods and/or drinks with minimal nutritional value per day (e.g. SSB, sweetened foods) than children from households receiving only WIC benefits. This layer of complexity has support in a former research study, which suggested that beneficiaries of SNAP and WIC were more likely to use their benefits to purchase SSB than beneficiaries of either programme alone $^{(19)}$. In the SNAP literature, a recent study comparing food consumption and programme participation found that women who were SNAP participants consumed $61 \%$ more SSB than women who were income-eligible but did not participate in the programme, suggesting that access to healthy foods simultaneously increased access to foods with minimal nutritional value ${ }^{(15)}$. A likely explanation for these patterns could be the higher price elasticity associated with having lower socio-economic status, a phenomenon that has been demonstrated in a number of obesity prevention and tobacco control efforts from the past $^{(38)}$.

\section{Limitations}

Although the analysis in the present study is unique in its evaluation of differential food consumption patterns among beneficiaries of dual SNAP and WIC, there are a number of limitations to this project. First, the cross-sectional sample lacks representation of only SNAP beneficiaries and low-income eligible non-participants of food assistance programmes. Second, because the SNAP beneficiaries were drawn from the larger WIC sample, there may be skewed exposure towards eating more healthful foods due to WIC's guidelines (i.e. restrictions to healthy food purchases and mandatory nutrition education). To disentangle this potential 'crossover' effect, future studies should consider comparing SNAP and WIC beneficiaries with only SNAP beneficiaries who are not exposed to the WIC guidelines. Third, the main and follow-up WIC surveys employed parent self-reports to assess child (beneficiary) outcomes. As a result, social desirability bias towards reporting healthier eating patterns may have occurred and is likely an unintended effect of the study design. Fourth, WIC participants who also receive SNAP may under-report their dual participation 
status, leading to under-representation of the dual SNAP and WIC group. Fifth, the conservative assumption that 'some but less than 1 full serving' equates to $0 \cdot 25$ servings may underestimate the actual fraction of servings consumed; however, this possibility is attenuated by the negligible number of survey participants who responded with this option. Sixth, because only complete cases were included in regression analyses, there is potential selection bias if those who did not complete the survey interview were not missing completely at random. This can be of particular concern due to potential reduction of cell counts; however, the present study did not find significantly reduced individual cell counts for variables in the regression model following list-wise deletion. Seventh, there may be reporting inaccuracies from parents who send their children to daycare and are thus unaware of their child's diet throughout the day. This can lead to recall bias, which may skew the association between self-reported food intake and programme participation. Finally, due to the nature of the survey design, the point estimates that were generated could not be used to distinguish temporality for the associations described.

\section{Conclusions}

Study findings suggest an interesting quandary for administrators of nutrition assistance programmes in the USA, as increased access to better nutrition also leads to increased access to foods with minimal nutritional value. As both SNAP and WIC have similar goals to increase food access and promote health for low-income populations, future programme planning should take these findings into consideration and use them to guide programme improvement efforts for these two food assistance programmes. Complementary nutrition education efforts across the two programmes may help programme enrollees maximize healthful food purchases with SNAP dollars.

\section{Acknowledgements}

Acknowledgements: The authors thank Mirna Ponce Jewell in the Division of Chronic Disease and Injury Prevention at the Los Angeles County Department of Public Health for her technical assistance with data analysis. Financial support: The project was supported in part by First 5 LA through a research partnership with Public Health Foundation Enterprises WIC Program (PHFE-WIC) and the California Department of Public Health's Nutrition Education and Obesity Prevention (NEOP) Project (contract number 12-10170) with funding from the US Department of Agriculture. Conflict of interest: No conflicts of interest or financial disclosures were reported by the authors. Disclaimer: The findings and discussions in this paper do not necessarily reflect the views or position of the Los Angeles County Department of Public Health; the Public Health Foundation Enterprises WIC Program; the US Department of Agriculture; the California Department of Public Health; or any other organization mentioned in the article. Authorship: J.L., S.E.W. and T.K. conceptualized the analysis plan. L.J. and B.R. assisted with the interpretation of the data and synthesis of the literature. All authors collaborated in the formulation of the paper's overall strategic direction and provided iterative input in the writing of the manuscript. Ethics of buman subject participation: The surveys received approval from the Independent Review Consulting Institutional Review Board (for PHFE-WIC) and the Los Angeles County Department of Public Health (for NEOP).

\section{References}

1. Nord M (2009) Food Insecurity in Households with Children: Prevalence, Severity, and Housebold Characteristics. Economic Information Bulletin no. EIB-56. Washington, DC: US Department of Agriculture, Economic Research Service; available at http://files.eric.ed.gov/ fulltext/ED508211.pdf

2. Mabli J \& Ohls J (2015) Supplemental Nutrition Assistance Program participation is associated with an increase in household food security in a national evaluation. $J$ Nutr 145, 344-351.

3. US Department of Agriculture, Food and Nutrition Service (2014) Supplemental Nutrition Assistance Program (SNAP). http://www.fns.usda.gov/snap/supplemental-nutrition-assis tance-program-snap (accessed May 2015).

4. Falk G \& Aussenberg RA (2014) The Supplemental Nutrition Assistance Program (SNAP): Categorical Eligibility. https:// www.fas.org/sgp/crs/misc/R42054.pdf (accessed August 2015).

5. Food Research and Action Center (2010) SNAP/Food Stamp Eligibility. http://frac.org/federal-foodnutrition-programs/ snapfood-stamps/eligibility/ (accessed May 2015).

6. US Department of Agriculture, Food and Nutrition Service (2014) Women, Infants, and Children (WIC). http://www. fns.usda.gov/wic/wic-benefits-and-services (accessed May 2015).

7. US Department of Agriculture, WIC Works Resource System (2015) WIC Program Nutrition Education Guidance. http://www.nal.usda.gov/wicworks/Learning_Center/ntred guidance.pdf (accessed September 2015).

8. Food Research and Action Center (2010) County-by-County Review of SNAP/Food Stamp Participation. http://frac.org/ wp-content/uploads/2010/07/ny_times_snap_poverty_form atted.pdf (accessed August 2015).

9. Nutrition Education and Obesity Prevention Branch (2013) Impact Outcome Evaluation Project. http://www.cdph.ca. gov/programs/cpns/Documents/FinalImpactEvaluation ReportFFY2013.pdf (accessed August 2015).

10. US Department of Agriculture, Food and Nutrition Service (2014) Supplemental Nutrition Assistance Program Education (SNAP-Ed). http://www.fns.usda.gov/snap/sup plemental-nutrition-assistance-program-education-snap-ed (accessed September 2015).

11. US Department of Agriculture, SNAP-Ed (2014) SNAP-Ed Strategies \& Interventions: An Obesity Prevention Toolkit for States. http://snap.nal.usda.gov/snap/SNAP-EdInterven tionsToolkit.pdf (accessed September 2015). 
12. US Department of Agriculture, Food and Nutrition Service (2012) Building a Healthy America: A Profile of the Supplemental Nutrition Assistance Program. http://www. fns.usda.gov/sites/default/files/BuildingHealthyAmerica.pdf (accessed June 2015).

13. Odoms-Young AM, Kong A, Schiffer LA et al. (2015) Impact of interventions to reduce sugar-sweetened beverage consumption in children and adults: a protocol for a systematic review and meta-analysis. Syst Rev 4, 17.

14. Emerson JS, Towns DR, Jones JL et al. (2015) Racial/ethnic and weight status differences in food preparation among WIC participants. J Health Care Poor Underserved 26, 335-344.

15. Leung CW, Ding EL, Catalano PJ et al. (2012) Dietary consumption and dietary quality of low-income adults in the Supplemental Nutrition Assistance Program. Am J Clin Nutr 96, 977-988

16. Barragan N, Gase L, Butler R et al. (2015) Self-reported use of supplemental nutrition assistance program benefits to purchase soda in a public health center population: Los Angeles County, California, 2012. Public Health Rep 130, 207-212.

17. Leung CW \& Villamor E (2011) Is participation in food and income assistance programmes associated with obesity in California adults? Results from a state-wide survey. Public Health Nutr 14, 645-652.

18. Larson NI \& Story MT (2011) Food insecurity and weight status among US children and families: a review of the literature. Am J Prev Med 40, 166-173.

19. Andreyeva T, Luedicke J, Henderson KE et al. (2012) Grocery store beverage choices by participants in federal food assistance and nutrition programs. Am J Prev Med 43, 411-418.

20. Kuo-Liang C, Zastrow M, Zdorovtsov C et al. (2015) Do SNAP and WIC programs encourage more fruit and vegetable intake? A household survey in the Northern Great Plains. J Fam Econ 36, 477-490.

21. Public Health Foundation Enterprises WIC Program (2014) The Los Angeles County WIC Survey. http://www. lawicdata.org/survey/ (accessed July 2015).

22. Francou A, Hebel P, Braesco V et al. (2015) Consumption patterns of fruit and vegetable juices and dietary nutrient density among French children and adults. Nutrients 7, 6073-6087.

23. Neumark-Sztainer D, Wall M, Perry C et al. (2003) Correlates of fruit and vegetable consumption among adolescents: findings from Project EAT. Prev Med 37, 198-208.

24. Field Research Corporation (2014) The Survey Methods Used to Complete a Follow-up Survey of SNAP-Eligible WIC Beneficiaries in Los Angeles County. San Francisco, CA: Field Research Corporation.

25. Los Angeles County Department of Public Health (2005) The Los Angeles County Health Survey. http://www.publichealth. lacounty.gov/ha/hasurveyintro.htm (accessed August 2015).
26. Field Research Corporation (2014) A Summary of the Survey Methods Used to Conduct the 2014 WIC Participants Survey. San Francisco, CA: Field Research Corporation.

27. US Department of Agriculture, Economic Research Service (2012) US Household Food Security Survey Module: Six-Item Short Form. http://www.ers.usda.gov/datafiles/ Food_Security_in_the_United_States/Food_Security_Survey_ Modules/short2012.pdf (accessed September 2015).

28. Schneider A, Hommel G, Blettner M et al. (2010) Linear regression analysis: part 14 of a series on evaluation of scientific publications. Dtsch Arztebl Int 107, 776-782; available at http://www.aerzteblatt.de/pdf.asp?id=79009

29. Lorson BA, Melgar-Quinonez HR \& Taylor CA (2009) Correlates of fruit and vegetable consumptions in US children. J Am Diet Assoc 109, 474-478.

30. Siega-Riz AM, Kranz S, Blanchette D et al. (2004) The effect of participation in the WIC program on preschoolers' diets. J Pediatr 144, 229-234.

31. Molitor F, Sugerman S, Yu H et al. (2015) Reach of Supplemental Nutrition Assistance Program-Education (SNAP-Ed) interventions and nutrition and physical activityrelated outcomes, California, 2011-2012. Prev Chronic Dis 12, E33.

32. Shenkin JD \& Jacobson MF (2010) Using the food stamp program and other methods to promote healthy diets for low-income consumers. Am J Public Health 100, 1562-1564.

33. Andreyeva T, Long MW \& Brownell KD (2009) The impact of food prices on consumption: a systematic review of research on the price elasticity of demand for food. Am J Public Health 100, 216-222.

34. Food Research and Action Center (2015) Why Low-Income and Food Insecure People are Vulnerable to Overweight and Obesity. http://frac.org/initiatives/hunger-and-obesity/ why-are-low-income-and-food-insecure-people-vulnerableto-obesity/ (accessed October 2015).

35. Cassady D, Jetter KM \& Culp J (2007) Is price a barrier to eating more fruits and vegetables for low-income families? J Am Diet Assoc 107, 1909-1915.

36. Leone LA, Beth D, Ickes SB et al. (2012) Attitudes toward fruit and vegetable consumption and farmers' market usage among low-income North Carolinians. J Hunger Environ Nutr 7, 64-76.

37. Haynes-Maslow L, Parsons SE, Wheeler SB et al. (2011) A qualitative study of perceived barriers to fruit and vegetable consumption among low-income populations, North Carolina, 2011. Prev Chronic Dis 10, E34.

38. Townsend J, Roderick P \& Cooper J (1994) Cigarette smoking by socioeconomic group, sex, and age: effects of price, income, and health publicity. BMJ $\mathbf{3 0 9}$, 923-927. 